\title{
Experimental Evaluation of a Novel Backflush Controller for Immersed Membrane Systems
}

\author{
L. $\operatorname{Erdei}^{1}$, S. Vigneswaran ${ }^{2}$ \& \& P. J. Smith ${ }^{3}$ \\ ${ }^{1,2 \& 3}$ Faculty of Engineering, University of Technology, Sydney \\ P. O. Box 123, 1 Broadway, NSW 2007 \\ AUSTRALIA
}

\begin{abstract}
Membrane backflush with air or permeate is commonly employed to reduce fouling in immersed micro- and ultrafiltration (MF/UF). Membrane plants traditionally rely on simple timer control automation to initiate periodic backflushes for membrane cleaning. In this study, a closed-loop (feedback) type backflush controller was evaluated experimentally with a bench-scale hollow fibre immersed MF/UF membrane - flocculation hybrid system.

The controller showed an unexpected behaviour at low fouling rates, which was manifested in significantly varying filtration periods. The investigation identified the cause in the use of a constant stabilisation lag parameter. The role of this control parameter was to determine the reversible component of fouling in real-time, which is a difficult problem in transient flow conditions that characterise the start of filtration periods. The resulting error became significant at low fouling rates and impaired effective control.

Based on this insight, we propose alternative, more robust control parameters, which can provide improved backwash control solutions for MF/UF membrane applications.
\end{abstract}

Keywords: Immersed membrane, fouling, backflush, feedback control

\subsection{INTRODUCTION}

Low-pressure immersed (also called submersed) MF/UF membrane filtration is becoming increasingly popular in water and wastewater treatment. Such membrane plants offer reliability and simple operation at increasingly affordable costs. In membrane applications, one of the major technical problems is membrane fouling, which is a transient or irreversible loss of membrane productivity [1]. Permeate flux decreases in MF/UF processes due to the retained solids that accumulate on, and within the membrane, which increase resistance to flow. Build-up of rejected matter on the top surface of the membrane causes external fouling (cake formation); while deposition and adsorption of small particles or macromolecules within the pores of the membrane results in internal fouling.

Fouling can be abated by various techniques, such as feed pre-treatment, the use of additional force fields, system operation at low fluxes, the manipulation of hydrodynamic conditions, backwash with permeate and/or air, membrane relaxation, and preventive chemical treatment $[2,3]$. These

* Correspondence to: S. Vigneswaran (tel.: +61 295142641, fax: +61 295142633, email: s.vigneswaran@uts.edu.au) 
methods, often in combination, have been used with varying degrees of success. Although the rate of fouling might be significantly reduced, with the progress of filtration, the trans-membrane pressure (TMP) increases and exceeds a set limit. The fouled membrane must be taken off-line and thoroughly cleaned to restore permeability an operational hurdle, which is expensive, and reduces the membrane. Therefore, better understanding and control of fouling remains the key operating consideration and an important research aim.

Membrane plants often employ periodic backflushes (or backwashes) using permeate and cleaning chemicals. Backflush sequence most often are initiated by electronic timers, and rarely, by permeate flow integrators (volume meters). Fouling, by definition, is the increase of TMP in constant flux filtration. Thus, it is more logical to use TMP change as a control parameter for backflush initiation than timers that do not consider and cannot adapt to process changes, such as the increase of feed turbidity. In contrast, TMP monitoring can detect the resulting increase and launch backflushes and if the change is unexpectedly high, also alarm the plant operator

This manuscript presents results of an experimental evaluation of a novel backwash control system that uses TMP feedback to control membrane backwash. The controller was partly described in earlier publications $[4,5]$, and its essential characteristics are briefly summarised below.

\subsection{THEORY}

Membrane filtration involving hollow fibre immersed membranes is typically cyclic, comprisin filtration and backflush periods. During permeate production, the total TMP increases as schematically shown in Figure 1. The increase of TMP is dependent on multiple and often complex factors, such as the imposed permeate flux, temperature, feed composition, and membrane material properties.

The increase of TMP is counteracted with periodic backflushes. However, this method of membrane cleaning can remove only the reversible component of foulant layers. The irreversible foulan component eventually must be removed using intensive chemical cleaning methods. The irreversible

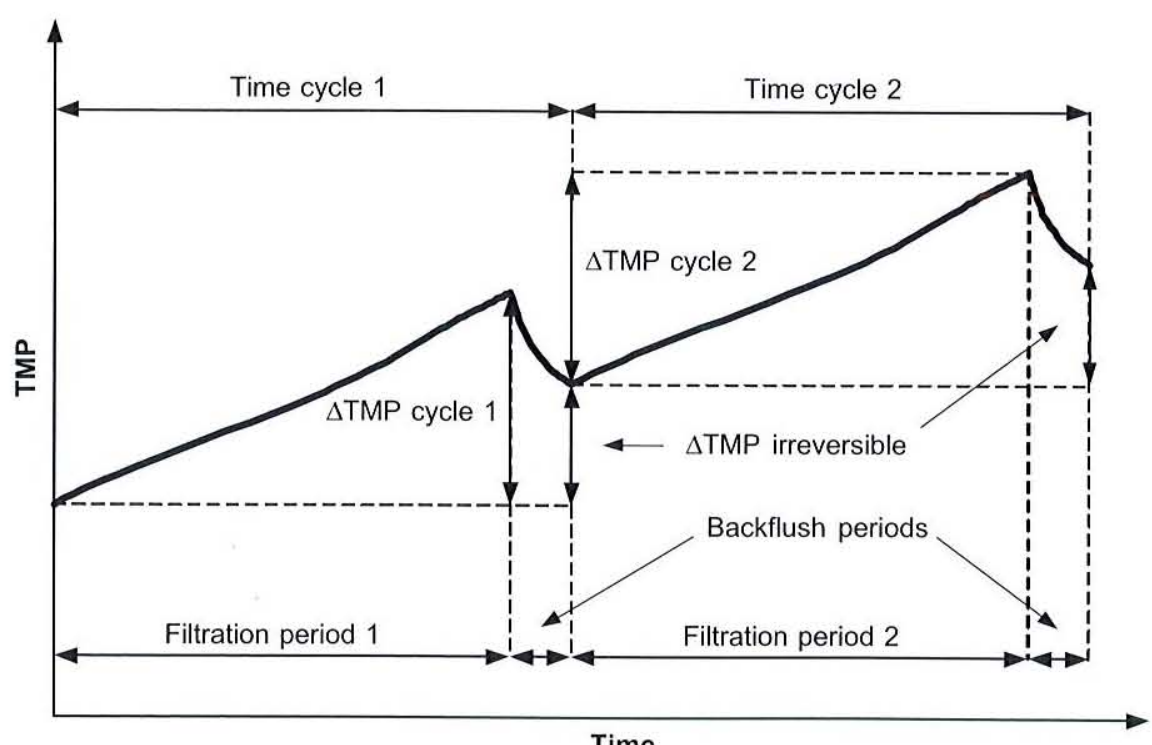

Time

Figure 1 Generic profile of TMP for periodic permeate production and backwash cycles part of fouling is dependent on the internal clogging of the membrane, the properties of the cake layer formed on the membrane surface, the duration and intensity of backflushes, and most importantly, the permeate volume produced, which is proportional to filtration time for constant flux. The increase the filtration periods and TMP may lead to cake layer compaction, which promotes the effective of tilion pering transition of revible fouling componet in be alleviated by limiting the total TMP increase during filtration periods. If the filtration periods are too short, however, both permeate and energy is wasted for backwashing, to decrease the net productivity of the plant.

\subsection{Process Control System}

Control systems supervise the operation of larger systems to ensure that overall responses approximate commanded behaviour [6]. The aim of a controller is to maintain a desired state of a process (also termed plant). Figure 2 shows the block diagram of a simple control system, which is called an openlop system becess output. A relevant example of open-loop control is a conventional timer that launches membrane backwash sequences periodically.

In contrast to open-loop systems, closed-loop control systems monitor the process output and use the obtained information to correct for disturbances (Figure 3)

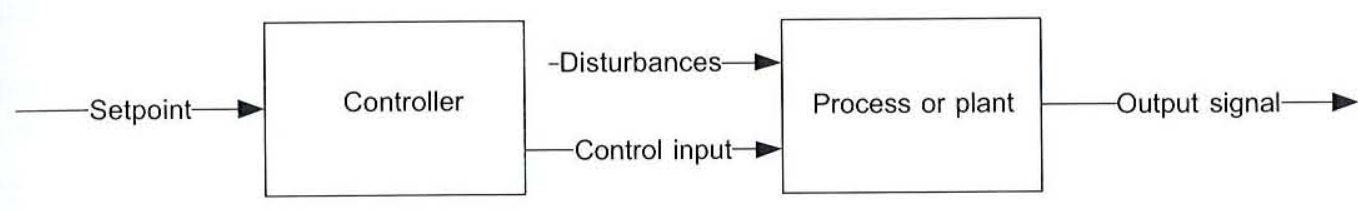

Figure 2 Open-loop control system

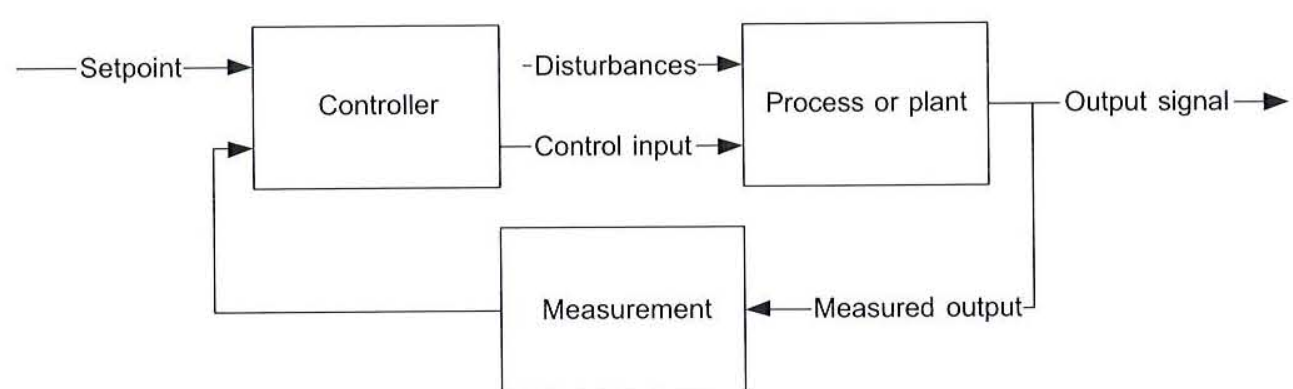

Figure 3 Closed-loop control system

Feedback controllers have at least one sensor to measure the parameter to be controlled, and an actuator to change the system's state in a way that affects the output signal. A simple example of a closed-loop control system is an electric heater that uses a thermostat to actuate a heating element to achieve a desired temperature (termed setpoint or reference signal). In a simple implementation

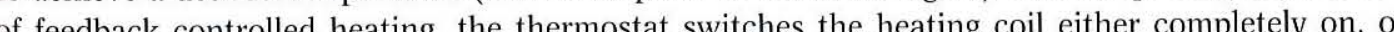
of feed con con completely off, thus an overshoot and undershoot of the controlled temperature is expected. A more refined control implementation would change the energy emitted by the heating element, depending on the difference (error signal) between the reference signal and the actual temperature (output 
signal). In many industrial applications, controllers compute the derivative and the integral of the error signal over time (PID controllers). The extra control terms improve response time, stability, and are able to reduce over and undershooting. However, the use of feedback alone does not guarante that the control will be stable, effective and reliable. To ensure high performance and robustness, control systems must be carefully designed, implemented, tuned, and thoroughly tested for all possible operating conditions.

The above considerations led to the design of a novel control system, which uses two parameters and feedback to limit the TMP increase to an optimum, predetermined value in each filtration period.

\subsection{Pressure Increment Control of Backflush Sequences}

The control system envisaged by Smith et al. [5] is a feedback type controller to start and stop periodic membrane backflushes. Since this controller attempts to maintain a desired TMP increment in each filtration cycle, in this study is termed a pressure increment controller (PIC). The controller requires the pre-selection of two parameters that are derived from prior membrane filtration experiments under timer control (TC). One control parameter is a system dependent stabilisation time (lag), another is the desired TMP increment value. Backflush duration and intensity are extra control parameters that were not in the scope of this investigation. The operational principle of the controller is explained with the help of Figure 4, noting that in all figures increasing TMP values indicate increasing vacuum.

At the end of a backflush event, the backwash valve closes and suction is applied to the membrane to produce permeate. For a relatively short interval (Ts) the TMP increases rapidly, along with the flow rate. Once the flow becomes steady, the further development of TMP is determined by fouling starting from $\mathrm{A}$. The PIC system uses the $\mathrm{S}$ setpoint to calculate the $\mathrm{TM}_{\mathrm{B}}=\mathrm{TMP}_{\mathrm{Ts}}+\mathrm{S}$ value when Ts time is elapsed, and from that moment compares this sum with measured (actual) TMP values to calculate the error signal every 2 seconds. The error signal decreases with increasing TMP, and when it becomes zero at B, the controller triggers the next backflush. The same control sequence is repeated

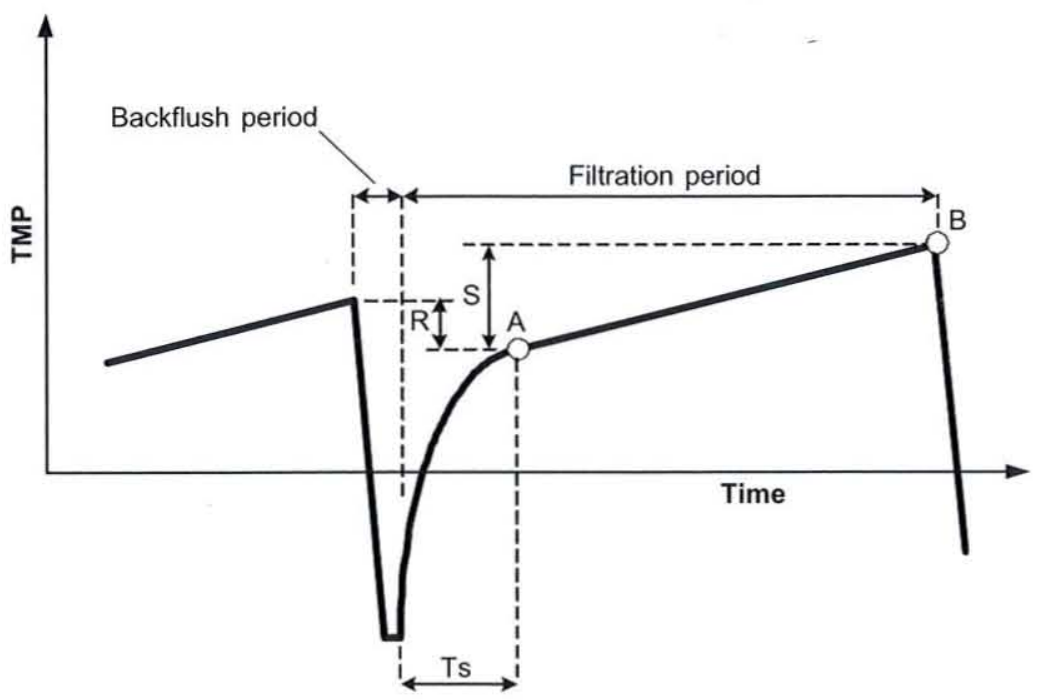

Figure 4 Parameters used by the pressure increment controlle until the TMP reaches an allowed maximum, after which the process is stopped for comprehensive chemical membrane cleaning.

Thus, in this feedback control system S is the desired TMP increment, and the measured TMP provides feedback to calculate the error signal. Although the setpoint is constant, its offset, which is equal to the instantaneous TMP at the lapse of Ts, does increase in each filtration period. The reversible fouling component $\mathrm{R}$ characterises the efficacy of the last backwash. In summary, the PIC implementation uses two pre-determined constant parameters and variable TMP feedback signal, whereas the common TC (open-loop control) requires a single constant, the imposed filtration period.

The PIC controller was tested with immersed membrane - PAC adsorption hybrid systems at laboratory and pilot plant scales $[4,5]$. It was claimed to minimise membrane fouling and maximise the productivity under those experimental conditions, by automatically optimising backflush initiations.

\subsection{METHODS}

Figure 5 shows the schematic diagram of the laboratory-scale immersed membrane flocculation hybrid system used in our experiments. Synthetic wastewater [7] was pumped into the membrane tank (6 $\mathrm{L}$ active volume) at constant flow rates. In-line coagulation was achieved by continuously pumping reagent grade ferric chloride or aluminium sulphate solution into the feed line, using a tee fitting and a $3 \mathrm{~mm}$ internal diameter tube section to provide 60 seconds mixing time for coagulation. Compressed air was introduced for aeration $(10 \mathrm{~L} / \mathrm{min}$ rate $)$ via a porous hose placed at the bottom of the membrane tank. At start-up the content of the reactor was slug-dosed with the coagulant, and most importantly, the membrane was immersed only after 45 minutes flocculation time to begin filtration. In experiments with clarified feeds, wastewater was pre-treated in a separate container and the supernatant was used after two hours settling. The feed was forced through the membrane under pump suction and permeate was collected in a container, for backwash supply. In these experiments, a Mitsubishi hollow fibre membrane module was used (hydrophilic PE material, 0.1

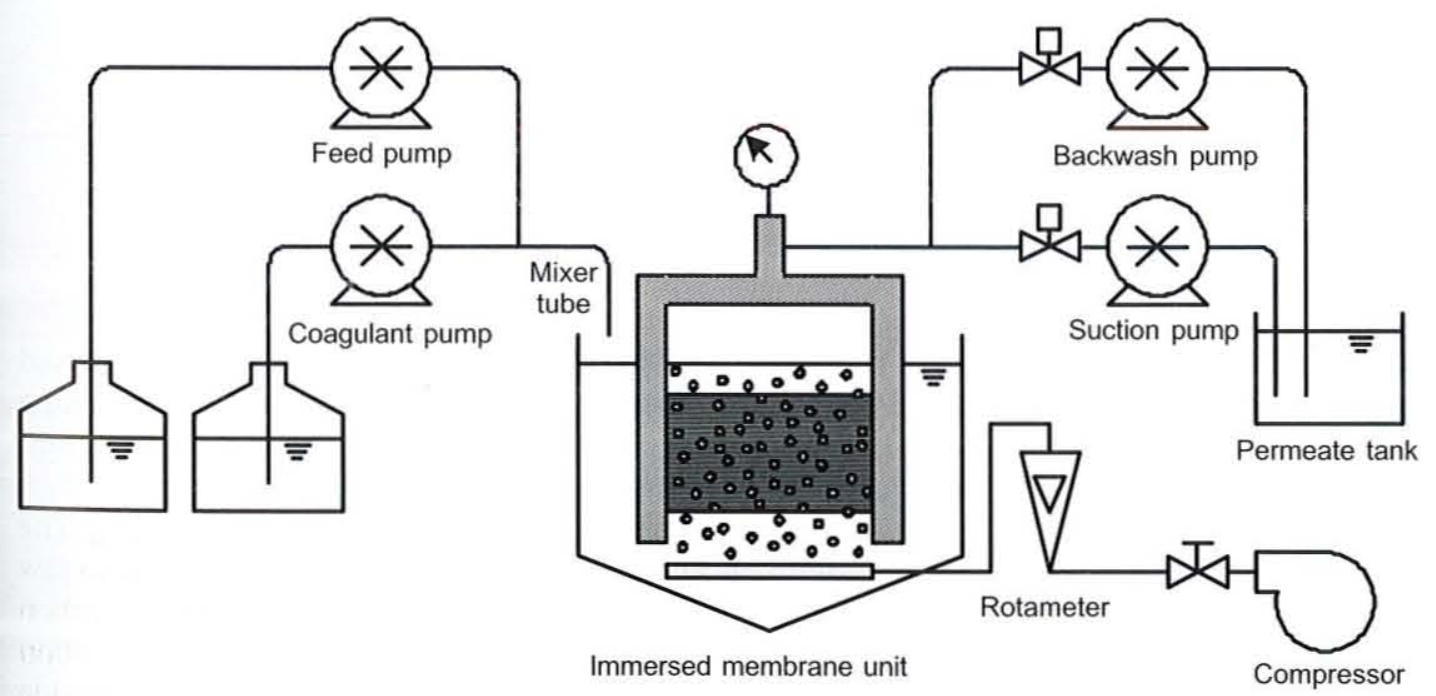

Figure 5 Schematic diagram of immersed membrane flocculation - hybrid system 
m pore size and $0.05 \mathrm{~m}^{2}$ total area). Membrane backflushes uniformly lasted for 30 seconds, at fluxes twice of the filtration fluxes. Optimum coagulant doses were pre-determined by jar tests. Permeate flows and membrane tank levels were constant in all experiments.

TMP development was measured with a Druck PTX 1400 type pressure transmitter every two seconds, and the data was normalised to $20^{\circ} \mathrm{C}$ temperature. An OMRON programmable logic controller (PLC) was used to actuate the solenoid valves and pumps, while a Citect supervisory control and data acquisition (SCADA) station logged data, system events, and displayed real-time and/or historical trends. Further details of the PLC and SCADA implementation are available in the literature [4].

Fouling rates were determined with post-processing from recorded data. For constant flux filtration and periodic backwashes, the rate of fouling can be determined from the increase of the irreversible TMP component over time, as illustrated in Figure 6.

The setpoints for PIC experiments were calculated from fouling rates obtained under TC. The values varied between 0.8 and $2.8 \mathrm{kPa}$ and were set individually for each experiment under PIC control. The stabilisation lags varied between 2 and 3 minutes in the preliminary timer-controlled experiments, thus the recommended average $(2.5$ minutes) was used in all PIC experiments.

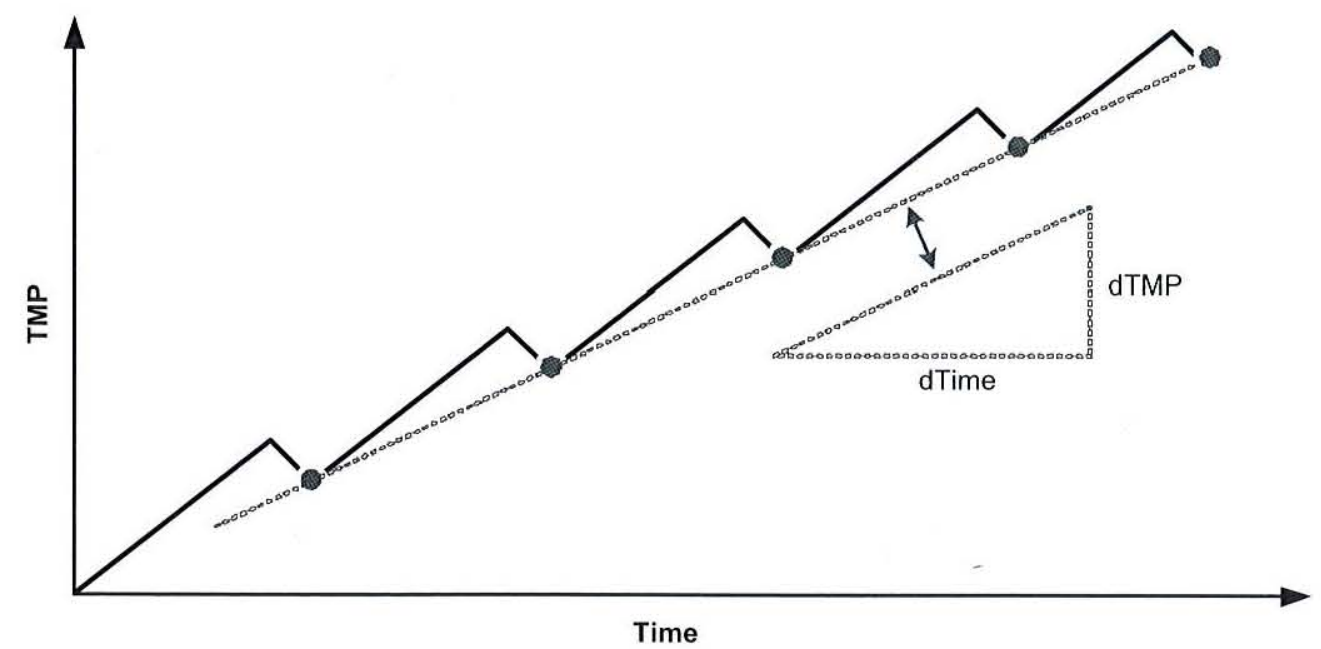

Figure 6 Determination of the rate of fouling using historical data

\subsection{RESULTS AND DISCUSSION}

In previous projects and experiments involving relatively high fouling rates [4, 5], the PIC showed clear and significant advantage over TC. Since the major aims of feed pre-treatment are maximum fouling reduction and foulant removal, the present experiments focused on low fouling conditions which occurred at optimum or near-optimum coagulant doses.

Feed flocculation pre-treatment was found to be very effective to reduce membrane fouling. The optimum dosage of ferric chloride $(22.38 \mathrm{mg} / \mathrm{L}$ as $\mathrm{Fe})$ reduced the rate of fouling compared to raw feed filtration nearly 20 times, and in the same time also ensured about 70\% dissolved organic carbon (DOC) removal. The fouling rate results obtained under timer control and fixed length filtration periods (30 minutes) are presented in Table 1, where all but one experiment involved ferric chloride coagulant.
Table 1 Summary of fouling rates under open-loop control

\begin{tabular}{cccc}
$\begin{array}{c}\text { Coagulant dose } \\
\mathbf{m g} / \mathbf{L}\end{array}$ & $\begin{array}{c}\text { Imposed flux } \\
\mathbf{L} / \mathbf{m}^{\mathbf{2}} \mathbf{h}\end{array}$ & $\begin{array}{c}\text { Fouling rate } \\
\mathbf{k P a} / \mathbf{h}\end{array}$ & Note \\
\hline 50 & 36 & 5.72 & Optimum alum dose \\
60 & 36 & 1.66 & \\
65 & 36 & 1.60 & Optimum dose \\
65 & 36 & 1.89 & Clarified feed \\
65 & 48 & 2.82 & \\
\hline
\end{tabular}

However, we found that in our experimental conditions the fouling rates under PIC were not smaller than under open-loop control, except for alum coagulant. Further, the filtration periods fluctuated substantially, which was contrary to design aims and expected control behaviour. This problem is explained in Figure 7, schematically showing the characteristic evolution of TMP in the experimental conditions.

It was reported by many researchers [8-10] that the initial, relatively short period of MF/UF membrane operation is controlled by rapid fouling due to pore blockage by small particles and/or macromolecular adsorption. Because of the rapid TMP increase, and the relatively small setpoint values used, in this short period the PIC would start frequent backflushes. We have found that backflushes in the early operating periods were ineffective. Therefore, it is logical to skip backflushes at the start of operation to save permeate, and optionally use this saving later when the cleaning process becomes more effective. This can be easily achieved, for example, by placing the controller in 'monitor-only' state in the first 20 minutes of operation.

Following the initial fouling period, the TMP evolution was typically linear to indicate cake filtration conditions. For linear TMP development the time cycles should be nearly equal $\left(T_{c 1} \approx T_{c 2} \approx T_{c 3}\right)$, since the setpoint is constant, and the (varying) reversible fouling component is only a small fraction of the total TMP. In contrast, actual data showed significantly varying cycle times. For example, Figure 9 shows the filtration periods for optimum ferric chloride coagulant dose at $48 \mathrm{~L} / \mathrm{m}^{2}$ imposed flux (last data row in Table 1)

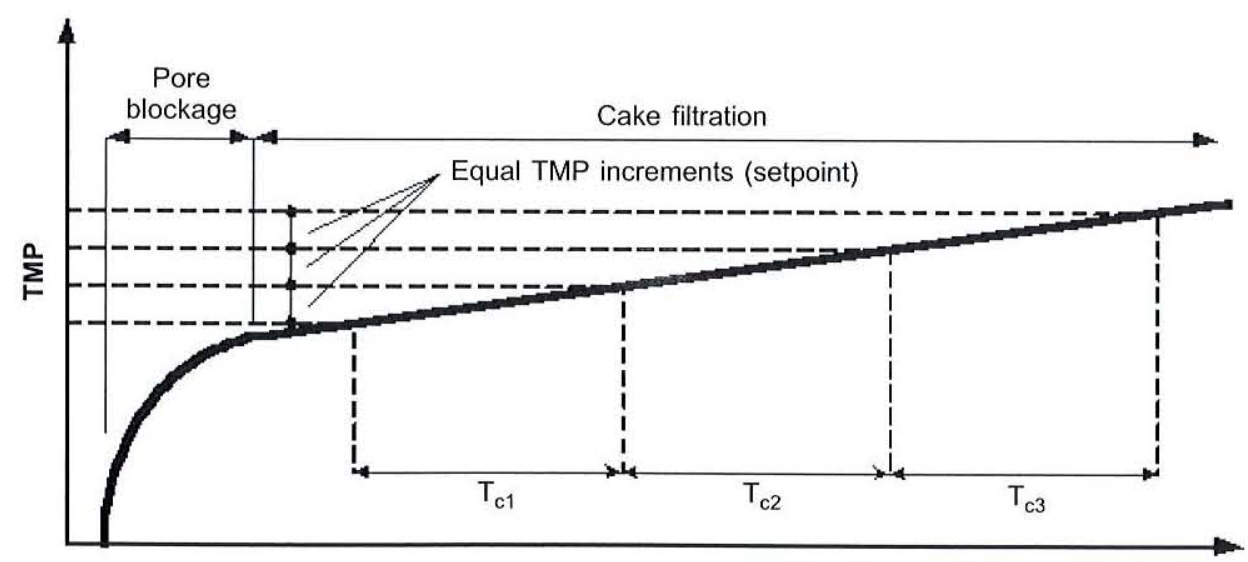

Time

Figure 7 Expected cycle times in cake filtration conditions 
In this experiment, the filtration periods evidently increased in the first half of operation and decreased in the second. The longest filtration period lasted for 42 minutes, more than twice the shortest one (18 minutes). Similar patterns were observed in other experiments, to indicate an impaired, yet consistent control behaviour.

Backwash optimisation studies showed that for a given backflush regime (pressure and duration), there exists an optimum filtration period for which the fouling rate becomes minimum $[5,11]$. On one hand, too short filtration periods do interfere with cake layer formation on the membrane, thus the cake cannot act as an effective pre-filter to the membrane. On the other hand, lengthy filtration periods decrease the porosity of the cake and increase its adhesiveness to the membrane. Therefore, substantially varying filtration periods under PIC, such as shown in Figure 8, are undesirable and suggest some subtle error in control.

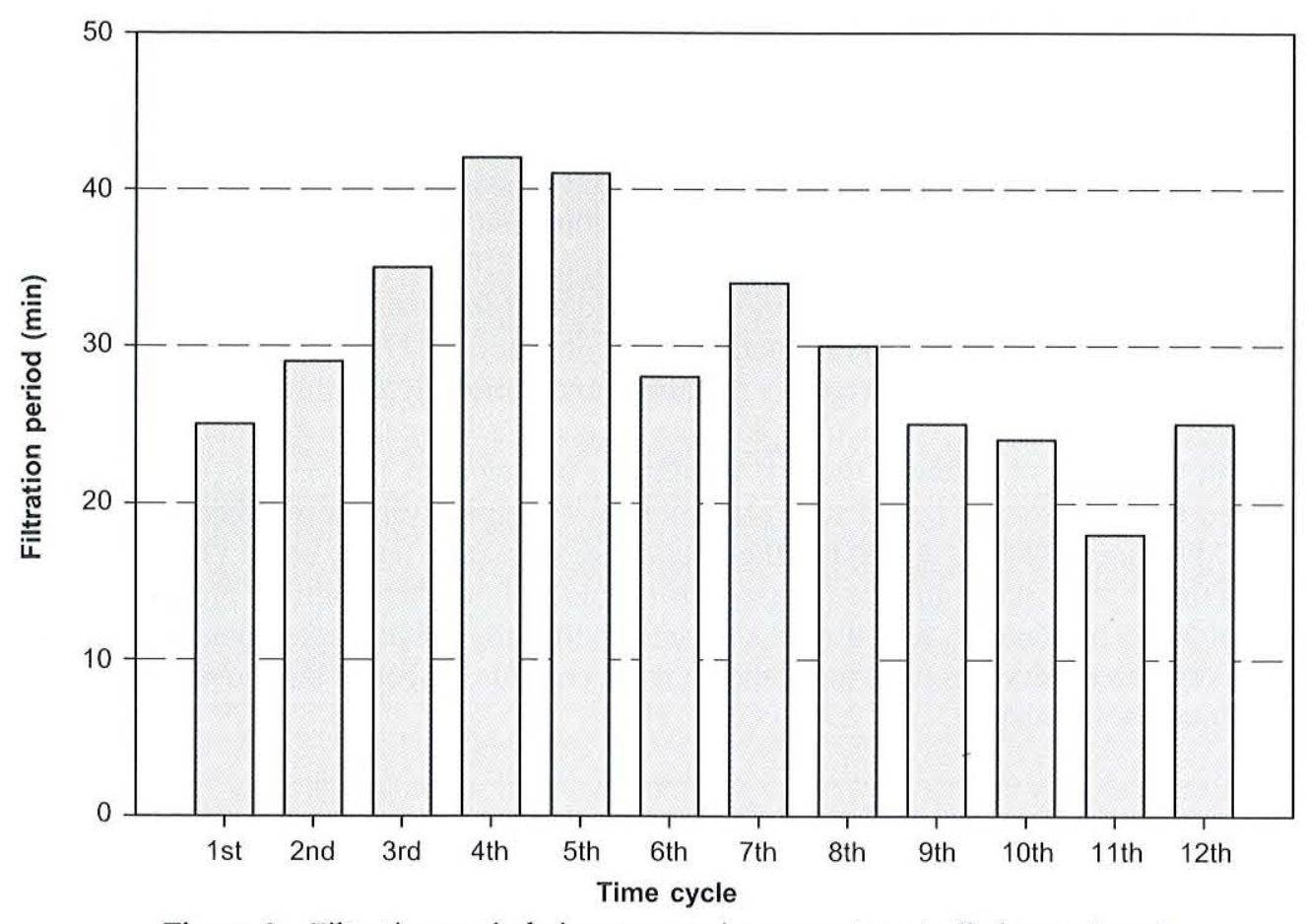

Figure 8 Filtration periods in pressure increment controlled experiment

\subsection{The Effect of Constant Stabilisation Lag Parameter}

The noted variation of filtration periods could be partly attributed to the increasing relative errors in TMP measurements at the prevailing low setpoint values. Measurements have random errors (noise) due to limited accuracy, and/or systematic errors, whether static (calibration) or time-dependent (shift). However, the control system used a software method to substantially reduce such errors, thus these could not explain the observed consistent and substantial variation of filtration periods. Therefore, the problem was likely caused by the flow stabilisation time parameter.

The experimental PIC implementation used fixed stabilisation lags to establish the onset of steady flow conditions. Preliminary experiments under open-loop control showed that stabilisation lags tended to increase with operating time, but in complex, non-monotonic manner. The use of an average lag value was a design decision, assuming that it can be tuned to suit various operational conditions The problem introduced with the use of a constant lag is explained in Figure 9.

Under perfect control the constant lag is equal to the real lag, therefore, the onset of steady flow at B is correctly established. With the progress of filtration the feedback signal increases, and the controller duly triggers the next backflush at $\mathrm{F}$.

Let us consider now imperfect control, and select a filtration cycle near the end of the experiment. In such later cycles the hydraulic resistance of the membrane system is bigger than it was earlier because of the increased irreversible part of fouling. Pump delivery rates decrease with increasing resistance, resulting in the slower stabilisation of flow and TMP (dotted curve). However, the PIC still uses the same Ts value, and regards $C$ as the offset (or base) of the setpoint. As Figure 9 shows, the determined instantaneous TMP value at $\mathrm{C}$ is smaller than the correct value belonging to $\mathrm{D}$, which will result in a shorter filtration period, stopped at $\mathrm{E}$.

Conversely, for an early filtration period in the same experiment the control lag tends to be longer than the actual lag, to result in an increased offset value and a corresponding longer filtration period. These considerations can logically explain the observed variations of filtration periods. The error caused by the constant lag is relatively small for high fouling rates (due to steeper TMP curves and bigger setpoints) and may be acceptable for some applications, but it derogated control performance in low fouling conditions.

The increase of system hydraulic resistance with operational time can explain the general increase of stabilisation lags. The noted complex variation of individual lag values can be attributed to various factors, such as the individually variable efficiency of backflushes, uneven cake re-formation on the membrane, and possible changes in cake morphology [12]. These factors can influence TMP evolution and individual lags in an unpredictable, stochastic manner, increase noise in the output signal, to aggravate or to reduce the offset error. This poses a difficult challenge to overcome, since the cycle offset value must be determined in real-time, using limited and noisy data. For past time cycles the same task is much less demanding because the complete TMP evolution is available.

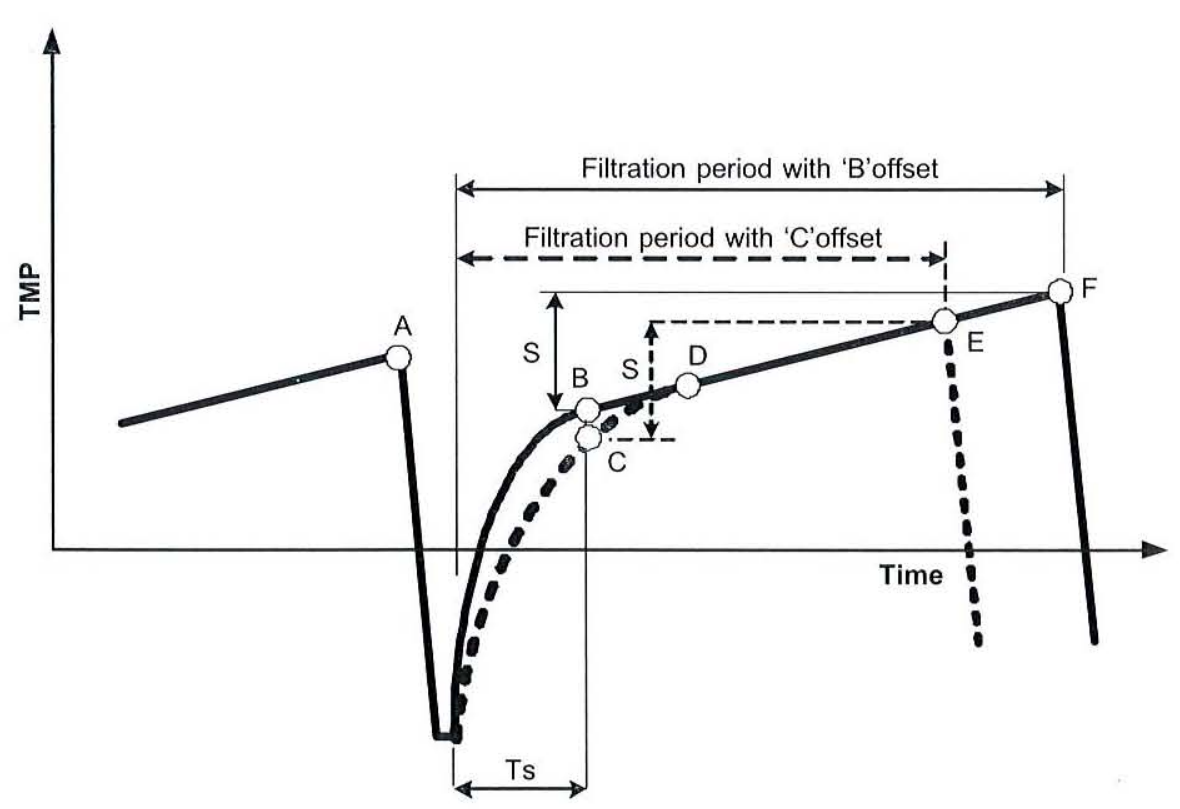

Figure 9 The effect of constant stabilisation lag on filtration periods 
These difficulties led us to reconsider the control on a more fundamental level, and find some other, more reliably and accurately determinable parameter.

\subsection{Improvements to Feedback Control}

The PIC implementation relied on the concept of reversible and irreversible fouling components, which is one possible way to characterise the efficacy of regular membrane backflushes. Here, we suggest an alternative concept which is more suitable for real-time control. The operational principle of the proposed control approach is explained with the help of Figure 10.

This new method characterises the efficacy of backflushes with a cycle interval and uses this parameter to control TMP development. In Figure 10, $\mathrm{TMP}_{\mathrm{A}}$ value is equal to the final TMP value of the previous filtration period, and $\mathrm{Tm}$ is the interval in which the feedback signal increases to $\mathrm{TMP}_{\mathrm{A}}$ value in the current filtration period. Filtration will be stopped for backwashing at $\mathrm{TMP}_{\mathrm{B}}=\mathrm{TMP}_{\mathrm{A}}+$ Sm feedback signal value, where $\mathrm{Sm}$ is the control setpoint.

The enhanced PIC system would operate quite similarly to the original PIC implementation, but relying on a simply determined and robust control interval. The longer this interval is, the lower the fouling rate is, and vice versa. The control interval can be related to backwash duration (or backwash volume) to characterise cleaning performance, and also to previous intervals. This way it becomes possible to use PID control, and to monitor fouling rate trends and adapt to process changes. For example, decreasing control intervals indicate increasing fouling rate. This trend may be counteracted with the increased duration and/or intensity of backflushes, and by dosing more chemical cleaning agent to the backwash permeate.

The (modified) TMP increment value $\mathrm{Sm}$ is determined the same way as $\mathrm{S}$ for the original PIC system, but the values will numerically differ. A further difference is that $\mathrm{Sm}$ is regarded a variable with a default value, rather than a constant. This does not make any essential operational difference in laboratory conditions, but it has advantages. For example, many full-scale plants do not have constant feed flow control. The membrane flux slightly and naturally declines during operation, and

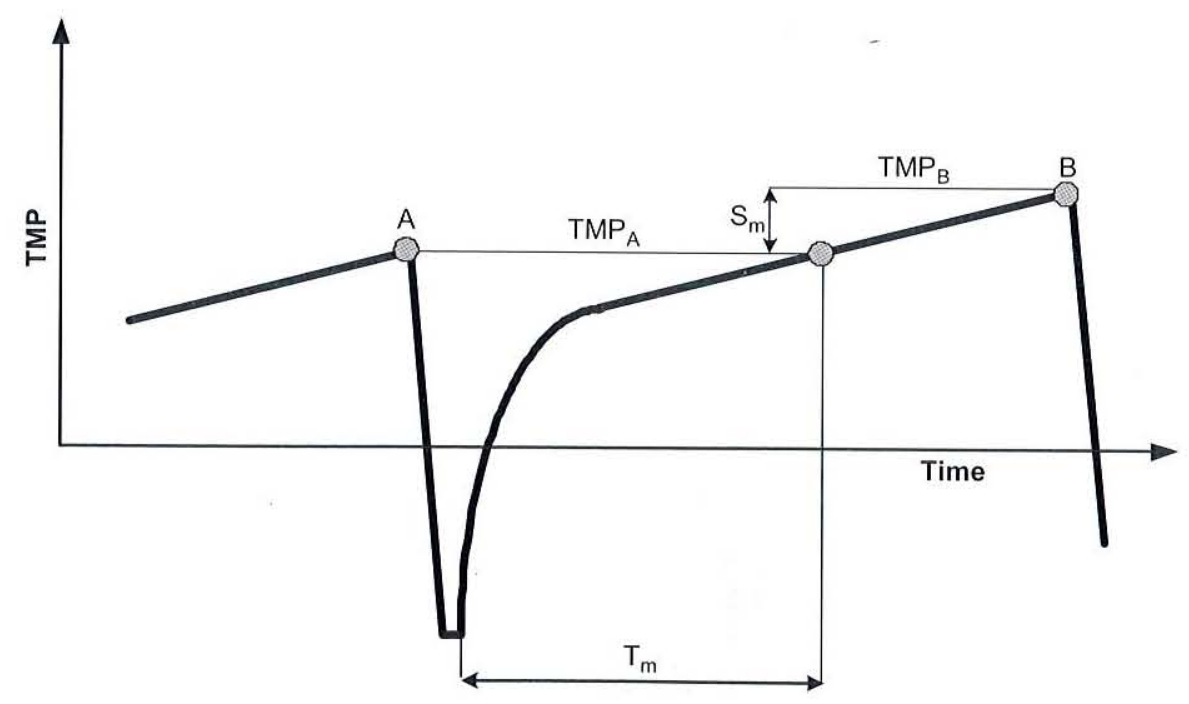

Figure 10 Parameters for improved control performance the feed pumps are adjusted periodically to meet the daily permeate production. A variable Sm parameter can take such common operational conditions into account. In any case, the use of variable control parameters does not add any complexity or cost to the PIC control, since it requires only a minor change in software.

A notable shortcoming of the evaluated PIC implementation was that it relied upon prior experimentation to obtain setpoint values. A more convenient and effective approach would be to enhance the controller with 'learning' ability, by adding artificial neural network and/or of fuzzy logic control components. Such components are normally provided in software and have become affordable for membrane filtration applications in recent years [13]. A controller enhanced with learning ability can find the setpoint parameter by itself, and autonomously adjust it for wanted optimums, such as minimised fouling or operational cost.

\subsection{CONCLUSIONS}

The evaluated closed-loop controller used for backflush initiation worked reliably and could monito the process in low fouling conditions. However, the investigation found unexpected and significant variations in filtration periods, which impaired control performance.

Analyses of historical data showed that measurement errors could partly contribute to cycle time variations, but such errors could not explain the observed substantial variation and pattern. The major cause of the problem was that the control system used a constant stabilisation period parameter With increasing operational time the TMP increased, which resulted in the general increase of rea stabilisation lags. A constant, average lag could determine the correct offset of the reference signal only in average conditions but caused over- and undershoot in the offset value in the beginning and end of experiments, respectively. Using a variable stabilisation lag is difficult to implement in any algorithm for real-time control, since the general trend of increasing stabilisation lags shows significant and unpredictable individual variations

We suggest an alternative approach to characterise the efficacy of individual backflushes for realtime control purposes. The new method uses two variable parameters, a TMP value and a cycle interval to determine the offset of reference signal. These parameters are robust, since always determined in stable flow conditions. Any current cycle interval can be related to those recorded in previous cycles, thus allowing the use of extra control terms required for PID control.

A further possible enhancement of the PIC controller is the addition of software components to provide a learning ability. An enhanced controller then can autonomously find and adjust its setpoint for optimised operation in varying process conditions.

\section{ACKNOWLEDGEMENTS}

The first author would like to thank the University of Technology, Sydney for his scholarship. This project was funded by DEST ISL and supported by EUROMBRA

\section{REFERENCES}

[1] Mallevialle, J., P. E. Odendal, and M. R. Wiesner. 1996. Water Treatment Membrane Processes $1^{\text {st }}$ edition New York: McGraw-Hill Professional. 
[2] Hilal, N., O. O. Ogunbiyi, N. J. Miles, and R. Nigmatullin. 2005. Methods Employed for Control of Fouling in MF and UF Membranes: A Comprehensive Review. Sep. Sci. Tech. 40(10): 1957-2005.

[3] Ma, H., L. F. Hakim, C. N. Bowman, and R. H. Davis. 2001. Factors Affecting Membrane Fouling Reduction by Surface Modification and Backpulsing. J. Membr. Sci. 189(2): 255-270.

[4] Smith, P. J., S. Vigneswaran, H. H. Ngo, H. Nguyen, and R. Ben-Aim. 2005. Application of an Automation System and a Supervisory Control and Data Acquisition (SCADA) System for the Optimal Operation of a Membrane Adsorption Hybrid System. IWA Conference on Instrumentation, Control and Automation. Busan, Korea. 2005: 201-208.

[5] Smith, P. J., S. Vigneswaran, H. H. Ngo, R. Ben-Aim, and H. Nguyen. 2006. A New Approach to Backwash Initiation in Membrane Systems. J. Membr. Sci. 278(1-2): 381-389.

[6] Nise, N. S. 2004. Control Systems Engineering. $4^{\text {th }}$ edition New York: John Wiley and Sons.

[7] Seo, G. T., S. Ohgaki, and Y. Suzuki. 1997. Sorption Characteristics of Biological Powdered Activated Carbon in BPAC-MF (Biological Powdered Activated Carbon-microfiltration) System for Refractory Organic Removal. Wat. Sci. Tech. 35(7): 163-170.

[8] Fane, A. G. and C. J. D. Fell. 1987. A Review of Fouling and Fouling Control in Ultrafiltration. Desal. 62: 117-136.

[9] Wakeman, R. J. and E. S. Tarleton. 1991. Colloidal Fouling of Microfiltration Membranes During the Treatment of Aqueous Feed Streams. Desal. 83: 35-52.

[10] Yuan, W., A. Kocic, and A. L. Zydney. 2002. Analysis of Humic Acid Fouling During Microfiltration Using a Pore Blockage-cake Filtration Model. J. Membr. Sci. 198(1): 51-62.

[11] Xu, Y., J. Dodds, and D. Leclerc. 1995. Optimization of a Discontinuous MicrofiltrationBackwash Process. Chem. Eng. J. 57(3): 247-251.

[12] Xu, W., S. Chellam, and D. A. Clifford. 2004. Indirect Evidence for Deposit Rearrangement During Dead-end Microfiltration of Iron Coagulated Suspensions. J. Membr. Sci. 239(2): 243-254.

[13] Cabassud, M., N. Delgrange-Vincent, C. Cabassud, L. Durand-Bourlier, and J. M. Laine. 2002. Neural Networks: A Tool to Improve UF Plant Productivity. Desal. 145: 223-231. 